\title{
Tumores de ovario. Exámenes de extensión. ¿una medida exagerada? Experiencia en el Hospital Universitario del Valle
}

\author{
José Saulo Torres R, MD*; Juan Pablo Suso, MD**; Eduardo Perea MD**; Luis Alberto Tafur, MD ***; \\ Raquel Fernández MD ****
}

\section{RESUMEN:}

Los exámenes de extensión ordenados a pacientes con masas pélvicas en forma rutinaria e indiscriminada conllevan a un gasto exagerado de los recursos, retardando el tratamiento primario y favoreciendo en ocasiones la deserción de pacientes.

PALABRAS CLAVES: Tumores de ovario, Exámenes de extensión, Beneficios.

\section{SUMMARY:}

Routine or randomly ordered extensions exams for patients with pelvic masses lead to an exaggerate use of resources, thus delaying primary treatment and sometimes, increasing patient's desertion rates.

KEY WORDS: Ovary Tumors, Additionall tests, Benefits.

El criterio Oncológico para el diagnóstico y tratamiento de los tumores de ovario exige una serie de exámenes llamados de extensión como son: endoscopia digestiva alta, rectoscopia, cistoscopia, urografía excretora, biopsia de endometrio, y marcadores tumorales $(1,2,3,4)$. Debido a que son requisitos indispensables en todos los centros Oncológicos, representan para la paciente erogaciones económicas importantes antes de la cirugía, los cuales en ocasiones no son realizados por escasez de recursos abandonando la institución, lo que conduce a ensombrecer su pronóstico. Teniendo en cuenta la existencia de criterios clínicos y ecográficos para predecir el comportamiento biológico y la extensión de la enfermedad podrían no solicitarse algunos de estos exámenes o al menos seleccionar los estrictamente necesarios para cada caso en particular.

Con el objetivo de determinar la poca utilidad, y el alto costo de los exámenes de extensión que se realizaron en las pacientes con sospecha clínica de tumor de ovario en el HUV de Cali, se revisaron los casos atendidos entre enero de 1994 y diciembre de 1996.

Jefe sección Ginecología Oncológica H.U.V. docente Ad-honorem Universidad del Valle Cali Colombia.

** Ginecólogos Oncólogos H.U.V. Docente Ad-honorem Universidad del Valle

*** Especialista Salud Pública. Profesor Titular Escuela de Salud Pública Universidad del Valle

**** Residente de tercer año Universidad Metropolitana de Barranquilla.

\section{MATERIALES Y METODOS:}

Se realizó la revişión de las historias clínicas de las pacientes operadas entre enero de 1994 y diciembre de 1996 con diagnóstico de tumor de ovario en la sección de Oncología Ginecológica del Hospital Universitario del

Tabla 1

INFORMES DE EXAMENES DE EXTENSION EN TUMORES BENIGNOS

\begin{tabular}{|l|cc|c|c|}
\hline & $\begin{array}{c}\text { Relacionado Normal } \\
\text { con } \\
\text { Diagnóstico }\end{array}$ & $\begin{array}{c}\text { Otros } \\
\text { Dx }\end{array}$ & Total \\
\hline Gastroscopia & 0 & 45 & 24 & 69 \\
\hline Rectoscopia & 13 & 64 & 0 & 77 \\
\hline Urografia & 10 & 63 & 0 & 73 \\
\hline Cistoscopia & 0 & 68 & 5 & 73 \\
\hline Legrado & 0 & 67 & 0 & 67 \\
\hline
\end{tabular}

Relacionado con el Diagnóstico significa todos aquellos informes que de alguna manera el tumor está influenciando en el resultado de alguna manera.

Tabla 2

INFORMES EXAMENES DE EXTENSION. TUMORES MALIGNOS

\begin{tabular}{|l|cc|c|c|}
\hline & $\begin{array}{c}\text { Relacionado } \\
\text { con } \\
\text { Diagnóstico }\end{array}$ & Normal & $\begin{array}{c}\text { Otros } \\
\text { Dx }\end{array}$ & Total \\
\hline Gastroscopia & 0 & 28 & $3 C$ & 58 \\
\hline Rectoscopia & 6 & 55 & 0 & 61 \\
\hline Urografia & 24 & 38 & 0 & 62 \\
\hline Cistoscopia & 1 & 50 & 5 & 56 \\
\hline Legrado & 2 & 38 & 0 & 40 \\
\hline
\end{tabular}

Relacionado con el Diagnóstico significa todos aquellos informes que de alguna manera el tumor está influenciando en el resultado de alguna manera, 
Valle, analizando los exámenes de extensión tomados antes de la cirugía clasificatoria.

La utilidad de los exámenes en relación con la predicción del comportamiento biológico y la extensión de la enfermedad se midió determinando si aportaron información básica o adicional a la obtenida con el examen físico y la ecografía pélvica inicial. Así mismo se cuantificaron los costos de estos exámenes. Las pacientes a quienes se les había definido conducta quirúrgica y estaban en lista de espera para cirugía, transcurridas tres semanas y no se reportaban al servicio eran contactadas por trabajo social y si los exámenes de extensión no se habían completado por falta de recursos eran llamadas para ser intervenidas. Esta es la razón por la que las pacientes no tienen todos los estudios de extensión completos.

\section{RESULTADOS}

De los 154 tumores de ovario operados de enero del 94 a diciembre del 96,68

eran malignos, 78 benignos y 8 de B.P.M. (B.P.M : Son tumores epiteliales con una baja tasa de crecimiento y un bajo potencial para invadir o metastatizar 5). Los exámenes de extensión que se realizaron al grupo de pacientes con tumores benignos y los de bajo potencial de malignidad no presentaron ningún hallazgo que modificara la conducta médico quirúrgica.

La gastroscopia y la rectoscopia previos a la cirugía diagnosticaron dos carcinomas gástricos y uno de recto respectivamente. Sin embargo tres casos de cáncer metastásicos a ovario no fueron diagnosticados con los exámenes de extensión.

La urografía excretora en el $50 \%$ de los casos fue normal, informó detalles inherentes a la compresión que causa el tumor en estructuras como vejiga y ureteres, hidronefrosis, y en un solo caso se sospecho invasión tumoral a la vejiga, la cual se confirmo por biopsia. Se diagnosticaron tres pacientes con sistema pielo-ureteral doble, y una agenesia de riñón derecho.

La cistoscopia en el $73 \%$ fue normal. Dentro de las alteraciones informadas lo más llamativo fue procesos inflamatorios, en ninguno de los casos se diagnosticó compromiso tumoral de la vejiga.

En cuanto a los legrados uterinos, una paciente (1\%) se diagnosticó adenoca mucinoso y en otra hiperplasia adenomatosa, el resto fueron negativos para malignidad (93\%).

En las pacientes con diagnóstico de tumores malignos, en los exámenes de extensión se detectaron dos Cánceres de estómago y uno de colon, las cuales no fueron operadas por el grupo de Oncología - Ginecológica, remitiéndose al servicio de gastroenterología.

\section{DISCUSION}

Se analizaron las historias clínicas de 154 pacientes con tumor de ovario a quienes se les realizaron exámenes de extensión, encontrándose que según los parámetros establecidos 68(44\%) estaban realmente "indicados" por tratarse de tumores malignos.

Los exámenes de extensión se han propuesto como base para aproximarse al diagnóstico del comportamien- to biológico y a la clasificación del Estadio clínico de la enfermedad. En la práctica oncológica quirúrgica el comportamiento biológico de la enfermedad y la estadificación del tumor se determinan mediante el estudio histológico de las piezas quirúrgicas (4), de tal manera que se le esta dando a los exámenes de extensión un atributo del que carecen.

Es importante precisar que, informes individuales como: compresión de uréteres, de vejiga, marcador Ca125 positivo, presencia de nódulo hepático, incluso ascitis etc. no son garantías que la paciente tenga un tumor maligno y mucho menos razonable es pretender una aproximación del Estadiaje clínico de la enfermedad.

La biopsia por congelación tiene sensibilidad cercana al 90\% (7), es esta la guía del cirujano en la intervención, al predecir el comportamiento biológico de la enfermedad, independiente de los exámenes de extensión.

La ecografía trans-vaginal podría racionalizar la utilización de los legrados endometriales recomendándoselo a todas las pacientes post-menopausicas con un endometrio mayor de $5 \mathrm{~mm}$. (6.)

Los hallazgos anteriores enfatizan que los únicos procedimientos que pueden predecir con alguna certeza el comportamiento biológico y el estadio clínico de la enfermedad son (4):

1. El Estudio histológico (Biopsia por congelación )

2. La cirugía clasificatoria.

El costo de los exámenes realizados a los pacientes osciló entre $\$ 632.000$ y $\$ 521,700$ (US\$ 632 y 521) dependiendo si se los practicará en forma privada o en una entidad del estado.

\begin{tabular}{|l|l|l|}
\hline Examen & Inst. Privada & H.U.V. \\
\hline Gastroscopia & $\$ 70.000$ & $\$ 91.200$ \\
\hline Rectoscopia & 70.000 & 74.000 \\
\hline Cistoscopia & 90.400 & 91.200 \\
\hline Orografia & 118.000 & 76.500 \\
\hline Bx. Endometrio & 140.000 & 45.000 \\
\hline Marcadores T & 143.800 & No se Procesa \\
\hline Total & $\$ 632.200$ & $\$ 521.700$ \\
\hline
\end{tabular}

Suponiendo que la totalidad de las pacientes se realizaran los exámenes, el gasto por este concepto en instituciones privadas implicaría la suma de $\$ 97.358 .800$ y el H.U.V de $\$ 80.341 .800$ (US $\$ 97.358$ y 80.341 ).

Para los tumores benignos el costo fue significativo si se tiene en cuenta que con este dinero el paciente cancelaba el procedimiento quirúrgico en una institución nivel III del Estado. A este costo se debe añadir lo relacionado con el tiempo que la paciente gasta realizándose los exámenes, y en muchas ocasiones determina que busque otra institución o en el peor de los casos no reciba el tratamiento por no presentar los resultados de los exámenes solicitados.

\section{CONCLUSIONES}

1. Los exámenes de extensión no aportan información adicional en la mayoría de los casos a la obtenida a través de una buena historia clínica, un adecuado examen físico, y la ecografía pélvica inicial., solo en $3(2 \%)$ de los pacientes se detectaron tumores primarios los cuales hicieron modificar la conducta médica. 
2. El costo de los exámenes de extensión y el tiempo invertido en su realización son un obstáculo para el acceso de las pacientes con diagnóstico presuntivo de tumor de ovario para la extirpación quirúrgica del tumor.

3. Se recomienda no solicitar exámenes de extensión en forma rutinaria en el estudio de las pacientes con diagnóstico presuntivo de cáncer de ovario. Cuando se soliciten deben ser específicos de acuerdo a la evaluación clínica y ecográfica inicial.

4. Ordenar exámenes innecesarios congestionan los servicios de diagnóstico, evitando que pacientes necesitados accedan a ellos prontamente.

5. Los únicos procedimientos que pueden predecir con alguna certeza el comportamiento biológico y el estadio clínico de la enfermedad son:

El Estudio histológico (Biopsia por congelación ) La cirugía clasificatoria.

6. Los tumores metastásicos a ovario detectados por los exámenes de extensión insospechados en el examen clínico son tan bajos (2\%) que no justifican su utilización masiva.

\section{RESUMEN}

Los denominados exámenes de extensión, han sido recomendados por distintos centros de enseñanza encargados de protocolizar el manejo de las pacientes oncológicas, con el fin de conocer él comportamiento biológico del tumor y aproximarse a una realidad de la extensión y el pronostico de la enfermedad.

En el caso de los tumores de ovario se recomiendan un sin número de exámenes como endoscopia digestiva alta, rectoscopia, cistoscopia, urografía excretora, biopsia de endometio y marcadores tumorales. Con el objeto de determinar la utilidad, y el costo de los exámenes de extensión que se realizaban en las pacientes con sospecha de tumor de ovario en el HUV de Cali, se presenta una revisión de los casos atendidos entre Enero de 1994 y Diciembre de 1996.

EL costo oscilo entre 80 y 98 millones de pesos(US\$ (80.000 y 98.000), los exámenes de extensión que se realizaron al grupo de pacientes con tumores benignos (78) y los de bajo potencial de malignidad (8) no presentaron ningún hallazgo que modificara la conducta médico quirúrgica y perfectamente habían podido ser omitidos. En las pacientes con diagnósticos de tumores malignos (68), se detectaron dos cánceres de estómago y uno de colon, las cuales se remitieron al servicio de gastroenterología.

Como conclusiones del estudio:

1. Es necesario idear un protocolo para seleccionar las verdaderas pacientes que se beneficiarían con los exámenes de extensión, donde los hallazgos encontrados verdaderamente modifiquen la conducta quirúrgica.

2. Los exámenes de extensión no aportan información adicional en la mayoría de los casos a la obtenida a través de una buena historia clínica, un adecuado examen físico, y la ecografía pélvica inicial.

3. El costo de los exámenes de extensión y el tiempo invertido en su realización son un obstáculo para que la paciente acceda prontamente a la extirpación quirúrgica del tumor.

4. Exámenes innecesarios (rectoscopias, cistoscopias, gastroscopias) congestionan los servicios de diagnóstico, retrasando estos en otros grupos de pacientes que verdaderamente los necesitan.

5. Los únicos procedimientos que pueden predecir con alguna certeza el comportamiento biológico y el estadio clínico de la enfermedad son: El Estudio histológico (Biopsia por congelación ) y la cirugía clasificatoria.

\section{BIBLIOGRAFIA}

1. Sección de Ginecología Oncológica Hospital Universitario del Valle. PROTOCOLOS DE MANEJO EN PATOLOGIA GINECO ONCOLOGICA. Cali Colombia 1995. ISBN 958-95736-0-6.

2. Instituto Nacional de Cancerología. PROTOCOLOS DE MANEJO. Bogotá, Colombia, 1988.

3. Celorio, J.A. Fundamentos de Oncología Ginecológica. Ediciones Diaz de Santos, S.A. 1986.

4. Disaia-Creasman. Oncología Ginecológica Clínica. Estadiaje. Pag: 675-81.Mosby/Doyma 1994. España.
5. Charles J Link Jr, Eddie Reed, Gisele Sarosy, Elise C Kohn. Borderline Ovarian Tumors, Review. The American Journal of Medicine. Volume 101. Agost 1996.Pag: 217-25.

6. Prompeler HJ y Col. Transvaginal sonography of miometrial invasión depth in endometrial cancer. Acta obst. Et gyn.scandinavica 73, 1994

7. Rose PG, Rubin RB, Nelson BE, Hunter RE y Reale FR. Accuracy of frozen-section (intraoperative consultation) diagnosis of ovarian tumors. Am J Obstet Ginecol 1994; 171: 823-826 